\title{
Parallel writing by local oxidation nanolithography with submicrometer resolution
}

\author{
Massimiliano Cavallini, Paolo Mei, and Fabio Biscarini ${ }^{\mathrm{a})}$ \\ CNR-ISMN Sez. Bologna, Via P. Gobetti 101, I-40129 Bologna, Italy \\ Ricardo García \\ Instituto de Microelectronica de Madrid, CSIC, Isaac Newton 8, 28760 Tres Cantos, Madrid, Spain
}

(Received 13 May 2003; accepted 20 October 2003)

\begin{abstract}
We demonstrate that the process of local oxidation of surfaces by atomic force microscopy (AFM) can be upscaled in a straightforward way by using a solid support with multiple protrusions as the cathode electrode. A metallized digital video disk DVD polymeric support has been used as a stamp to generate an array of features of variable length and $100 \mathrm{~nm}$ in width on a silicon surface covering a $5 \times 6 \mathrm{~mm}^{2}$ region. The parallel patterning process involves the formation of as many liquid bridges as there are protrusions in the stamp. The growth rate of the parallel local oxides is slightly smaller than the one obtained by AFM experiments. Nonetheless, results from AFM local oxidation experiments can be readily extended to parallel oxidation which in turn opens the possibility of patterning centimeter-square regions with $10 \mathrm{~nm}$ motives. () 2003 American Institute of Physics. [DOI: $10.1063 / 1.1633685]$
\end{abstract}

Local oxidation or nano-oxidation of metallic, organic, and semiconducting films by a conductive probe of an atomic force microscope (AFM) is emerging as a reliable and versatile method to fabricate nanometer-scale structures and devices. Nano-oxidation has been applied to yield patterns made of dots and lines of controlled size and positions that have been used to demonstrate bit writing with areal densities of about 1 terabit/in. ${ }^{2},{ }^{1,2}$ to generate sets of interdigitated lines with a periodicity of $13 \mathrm{~nm},{ }^{3}$ and to fabricate submicrometer gratings ${ }^{4}$ or optical waveguides with subwavelength cross sections. ${ }^{5}$ Local oxidation nanolithography has also been applied to fabricate several electronic devices with critical features in the nanometer range, such as singleelectron transistors, ${ }^{6}$ superconducting interference devices, ${ }^{7}$ or antidot lattices. ${ }^{8}$ Local oxidation has also been proven useful to seed defects acting as nuclei for crystallization or anchoring sites for receptors of biomolecules, ${ }^{9}$ to modify self-assembled monolayers, ${ }^{10}$ or to fabricate templates to orient growth of molecular thin films. ${ }^{11}$ Moreover, negative features, such as nanosized holes and trenches, can be achieved by anisotropic etching of the oxide layer. ${ }^{12}$

Local oxidation is compatible with ambient operation, microelectronic processing, and does not require masks or lift-off. In terms of resolution and writing speed, it is comparable to state-of-the-art electron-beam lithography, with the advantage of being more sustainable in terms of cost and being based on a widespread microscopy technique. The shortcomings arise from the strictly serial nature of the process that hinders its technological implications and by the limited area in which fabrication can be performed. The patterned area is limited by the maximum scan size of the piezoeletric scanner, usually in the $10^{-3} \mathrm{~mm}^{2}$ range. It would be therefore extremely desirable to transfer the same process developed for AFM into a parallel fabrication method ca-

a)Electronic mail: f.biscarini@ism.bo.cnr.it pable of similar performance in terms of positioning and feature definition, but covering centimeter-square regions. Although parallel local oxidation was demonstrated by using an array of $50 \times 1$ parallel cantilevers, ${ }^{13}$ current technical limitations in the fabrication and operation of arrays of parallel cantilevers have slowed down this approach.

Nano-oxidation has strong similarities with conventional anodic oxidation. The AFM tip is used as a cathode and the water meniscus formed between tip and surface is the source of the oxyanions species. ${ }^{1}$ The strong localization of the electrical field lines within the meniscus gives rise to a nanometer-sized oxide dot. ${ }^{1,14,15}$ The physics and chemistry of individual local oxidation processes are rather general and do not imply the use of an AFM tip as a cathode to perform the oxidation. Consequently, a setup can be envisioned in which the cathode is an array of metallic protrusions grown, deposited, or attached onto a solid support.

In this letter, we propose and demonstrate a method to pattern centimeter-square areas with submicrometer motives by using a parallel local oxidation scheme. We show that local oxidation can be effectively used with stamps brought into intimate contact with a silicon oxide surface. The stamps are metallic films grown onto a recorded digital video disk (DVD) polymeric support. By applying voltage pulses to the junction nanostructures of silicon oxide are grown onto the substrate over several square millimeters. The oxide structures replicate the position and periodicity of the protrusions of the stamp; however, their lateral width is about $100 \mathrm{~nm}$, smaller than the stamp features. Although the use of a stamp bears some resemblance to new lithographic methods for patterning soft materials, ${ }^{16-18}$ our approach is a resistless patterning process.

The experiment, schematically shown in Fig. 1, was performed using a home-built press able to hold substrates up to $6 \times 5 \mathrm{~cm}^{2}$. The plate holding the stamp slides along three cylinders that maintain it parallel to the substrate. A set of calibrated springs holds the plates against each other. Once 
(a)
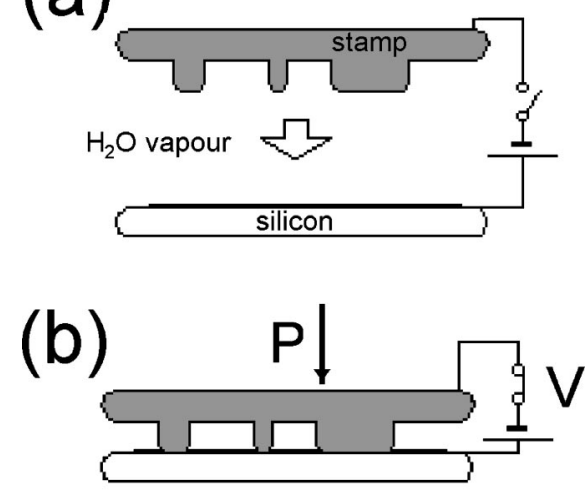

(c)

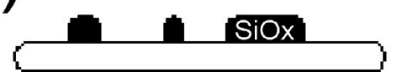

FIG. 1. (a)-(c) Schematic representation of the three main steps of parallel local oxidation.

in contact, a load force can be applied by tightening a micrometric screw. Here, we have applied load forces of approximately $2.5 \mathrm{~kg} / \mathrm{cm}^{2}$. The press is inserted in a chamber with controlled relative humidity by fluxing water-saturated nitrogen.

The stamp consists of a $1 \mathrm{~cm}^{2}$ piece of polycarbonate cut out of a recorded DVD, with an effective contact area of 5 $\times 6 \mathrm{~mm}^{2}$, and coated with $100 \mathrm{~nm} \mathrm{Au}$ film evaporated in high vacuum. Figure 2 shows the topography of the stamp's surface. Au film covers conformally the relieves of the DVD substrate, with 90-nm-high and $260 \mathrm{~nm}$-wide (full width at half-maximum) hillocks. Protrusions with variable lengths ranging from 350 to $1500 \mathrm{~nm}$ are present on the surface.

The substrate to be modified is a silicon (111) wafer covered with a native oxide layer of about $1 \mathrm{~nm}$. To transfer

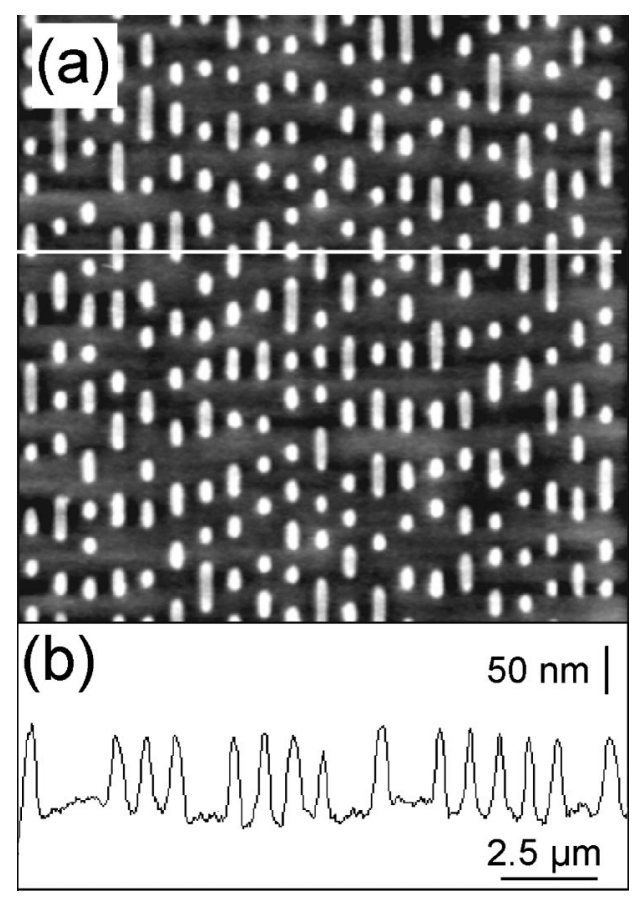

FIG. 2. (a) Topographic AFM image of the DVD stamp. (b) Cross section along the white line in (a). structures. Furthermore, the surface has been immersed in a
Downloaded 14 May 2010 to 161.111.235.252. Redistribution subject to AIP license or copyright; see http://apl.aip.org/apl/copyright.jsp

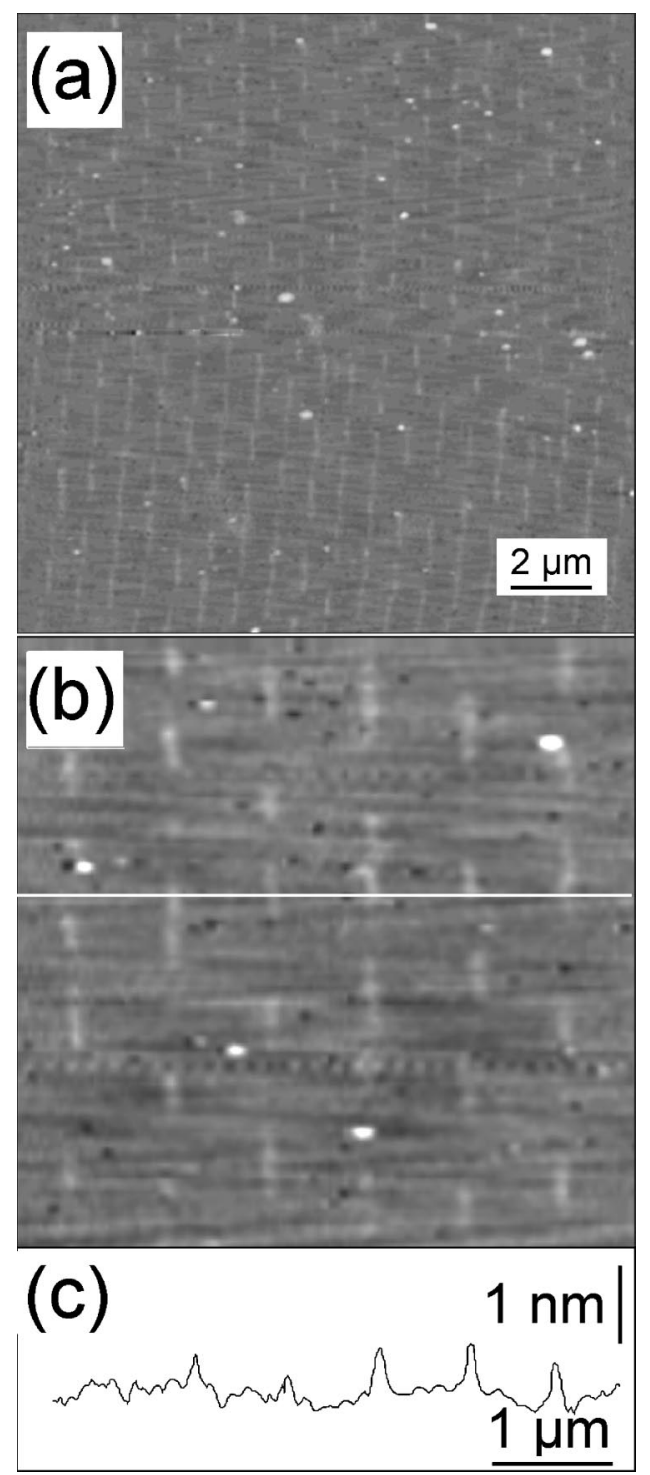

FIG. 3. Detailed view of the oxide structures fabricated on the silicon substrate by the application of a $15 \mathrm{~V}$ pulse for $15 \mathrm{~s}$. (a) AFM image of a 15 $\times 15 \mu \mathrm{m}^{2}$ area of the region in contact with the stamp that underwent local oxidation. Height range is 0 (black) to 2.8 (white) $\mathrm{nm}$. (b) $5 \times 5 \mu \mathrm{m}^{2}$ zoom of (a), height range is 0 (black) to 1.8 (white) $\mathrm{nm}$. (c) Cross section along the white line in (b).

the motives from the stamp to the substrate a 15 or $20 \mathrm{~V}$ bias silicon. Those parameters are comparable to the parameters used to perform local oxidation in contact and noncontact modes. $^{19}$

Figures 3(a) and 3(b) show the result over an area which is representative of the whole modified surface. The structures are $95 \pm 7 \mathrm{~nm}$ wide, with a variable length and a height of $0.7 \pm 0.2 \mathrm{~nm}$ with respect to the substrate baseline. The cross section in Fig. 3(c) along the line marked in (b) shows their height. These structures can be found all over the region in intimate contact with the stamp, and their characteristic sizes are rather homogeneous across the whole area. The distance between parallel lines is approximately $740 \mathrm{~nm}$, that matches perfectly that of the original DVD. No effect of increasing the load force is observed on the sample in terms of modification, displacement, or damage to the fabricated voltage for a time ranging between 20 and $180 \mathrm{~s}$ is applied to 


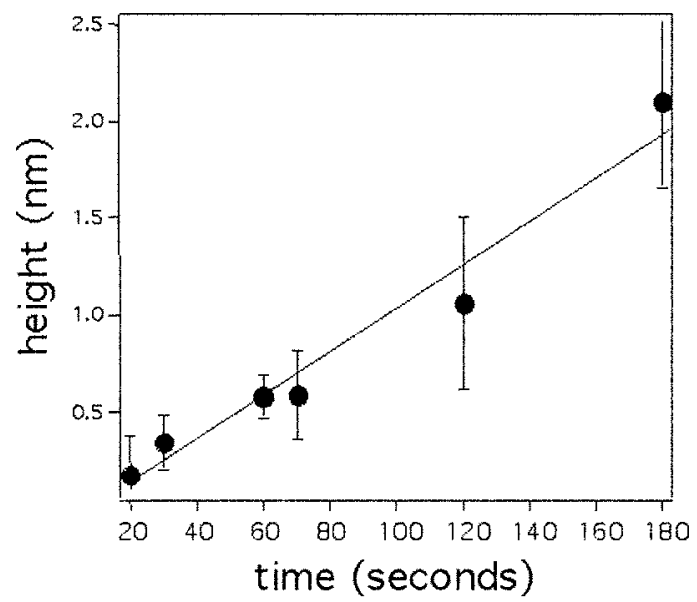

FIG. 4. Dependence of the height of oxide structures on bias voltage duration for an applied voltage of $20 \mathrm{~V}$. The continuous line is a guide to the eye.

$10 \%$ solution of hydrofluoric acid for $2 \mathrm{~min}$. The acid etches selectively silicon oxide versus silicon, leaving $\approx 1$-nm-deep trenches in correspondence of the fabricated structures. This is an indication that the process relevant to the creation of nanostructures is anodic oxidation through the formation of as many water bridges as there are protrusions in the stamp. Here, the formation of liquid bridges is driven by both the mechanical contact between the protrusions and the sample surface and the field-effect condensation of water molecules in the proximity of the protrusions. ${ }^{1,15}$ The electrical field would allow the formation of water meniscus even in the absence of mechanical contact.

Figure 3(b) shows a detailed view of the fabricated structures. The cross-section in Fig. 3(c) along the line marked in (b) reveals the height of the fabricated oxide structures. A statistics on the structures yields a width of 95 $\pm 7 \mathrm{~nm}$, a mean height of $0.7 \mathrm{~nm}$ and a roughness of less than $0.2 \mathrm{~nm}$ on top of the structures.

We have performed some preliminary experiments to determine the oxidation kinetics of parallel oxidation. Figure 4 shows the effect of the duration of the oxidation process on the height of the nanostructures. The oxide growth rate is slower than the growth rates obtained for $\mathrm{Si}(100)$ and $\mathrm{Si}(111)$ by AFM oxidation. ${ }^{19-21}$ This difference can be attributed to several factors. First, the effective electrical field underneath a protrusion should be smaller than in AFM local oxidation. This is because the curvature of the protrusion is smaller than that of an AFM tip. The smaller electrical field makes the oxidation diffusion barriers higher. Second, it has been observed that the kinetics of local oxidation depends on the AFM operating mode (contact or noncontact). Specifically, contact oxidation occurs at a lower rate with respect to noncontact oxidation. ${ }^{19}$ Third, the measurements of Fig. 4 are average values estimated over a statistical sample of more than 100 oxide structures made by independent protrusions, whereas in AFM there is only one protrusion (the tip).

Although in our experiment the stamp is pressed against the surface, it is reasonable to assume that only a fraction of the protrusions are in mechanical contact with the substrate. Nonetheless all the protrusions are transferred to the sub- strate. Figure 4 shows that the standard deviation ranges from $25 \%$ to $50 \%$ of the mean height, which suggests, indeed, a nonuniform protrusion-sample separation. The presence of a water meniscus bridging the protrusions and the substrate, implies that the voltage drops mostly within the native silicon oxide layer. This is because of the difference between water and silicon dioxide dielectric constants $(80$ versus 3.9). This may explain the ability to transfer all the protrusions despite of the variability of separation.

In conclusion, the process of local oxidation of surfaces by AFM can be upscaled by using a multiple source of protrusions. This has allowed the patterning of a $5 \times 6 \mathrm{~mm}^{2}$ region with submicrometer motives in $50 \mathrm{~ms}$. The patterning requires the formation of multiple and independent nanometer-sized water bridges. The body of AFM oxidation knowledge together with the use of a suitable design of the protrusions and the fine control of the distance between stamp and substrate with spacers ${ }^{18,22}$ allows us to predict that the fabrication of features with sub-10 $\mathrm{nm}$ resolution will be feasible with the same overall method. ${ }^{23}$

This work is supported by EC-Growth RTD Project MONA LISA G5RD-CT-2000-00349.

${ }^{1}$ R. Garcia, M. Calleja, and H. Roher, J. Appl. Phys. 86, 1898 (1999).

${ }^{2}$ E. B. Cooper, S. R. Manalis, H. Fang, H. Dai, K. Matsumoto, S. C. Minne, T. Hunt, and C. F. Quate, Appl. Phys. Lett. 75, 3566 (1999).

${ }^{3}$ M. Tello, F. Garcia, and R. Garcìa, J. Appl. Phys. 92, 4075 (2002).

${ }^{4}$ F. S. Chien, C.-L. Wu, Y.-C. Chou, T. T. Chen, S. Gwo, and W.-F. Hsieh, Appl. Phys. Lett. 75, 2429 (1999).

${ }^{5}$ T. Onuki, T. Tokizaki, Y. Watanabe, T. Tsuchiya, and T. Tani, Appl. Phys. Lett. 80, 4629 (2002).

${ }^{6}$ K. Matsumoto, Y. Gotoh, T. Maeda, J. A. Dagata, and J. S. Harris, Appl. Phys. Lett. 76, 239 (2000).

${ }^{7}$ V. Bouchiat, M. Faucher, C. Thirion, W. Wernsdorfer, T. Fournier, and B. Pannetier, Appl. Phys. Lett. 79, 123 (2001).

${ }^{8}$ A. Dorn, M. Sigrist, A. Fuhrer, T. Ihn, T. Heinzel, K. Ensslin, W. Wegscheider, and M. Bichler, Appl. Phys. Lett. 80, 252 (2002).

${ }^{9}$ T. Yoshinobu, J. Suzuki, H. Kurooka, W. C. Moon, and H. Iwasaki, Electrochim. Acta 48, 3131 (2003).

${ }^{10}$ R. Maoz, E. Frydman, S. R. Cohen, and J. Sagiv, Adv. Mater. (Weinheim, Ger.) 12, 725 (2000).

${ }^{11}$ F. Biscarini and R. Garcia, Patent No. 200300565, Spanish Patent Office (2003).

${ }^{12}$ F. S. Chien, Y.-C. Chou, T. T. Chen, W.-F. Hsieh, T. S. Chao, and S. Gwo, J. Appl. Phys. 89, 2465 (2001).

${ }^{13}$ S. C. Minne, J. D. Adams, G. Yaralioglu, S. R. Manalis, A. Atalar, and C. F. Quate, Appl. Phys. Lett. 73, 1742 (1998).

${ }^{14}$ H. Sugimura and N. Nakagiri, J. Appl. Phys. 34, 3406 (1995).

${ }^{15}$ M. Calleja, M. Tello, and R. Garcìa, J. Appl. Phys. 92, 5539 (2002); S. Gómez-Moñivas, J. J. Sáenz, M. Calleja, and R. Garcìa, Phys. Rev. Lett. 91, 056101 (2003).

${ }^{16}$ H. O. Jacobs and G. M. Whitesides, Science 291, 1763 (2001).

${ }^{17}$ E. Schäffer, T. Thurn-Albrecht, T. P. Russel, and U. Steiner, Nature (London) 403, 874 (2000).

${ }^{18}$ S. Chou, L. Zhuang, and L. Guo, Appl. Phys. Lett. 75, 1004 (1999).

${ }^{19}$ M. Tello and R. Garcìa, Appl. Phys. Lett. 79, 424 (2001).

${ }^{20}$ J. A. Dagata, F. Pérez-Murano, G. Abadal, K. Morimoto, T. Inoue, J. Itoh, and H. Yokoyama, Appl. Phys. Lett. 76, 2710 (2000).

${ }^{21}$ T. Teuschler, K. Mahr, S. Miyazaki, M. Hundhausen, and L. Ley, Appl. Phys. Lett. 67, 3144 (1995).

${ }^{22}$ M. Cavallini and F. Biscarini, Nano Lett. 3, 1269 (2003).

${ }^{23}$ As we were completing this work, we have become aware of a work reporting the transfer of micrometric patterns on a rigid metallic object to a highly ordered organosilane monolayer on silicon upon the application of a voltage between the object and the silicon substrate; S. Hoeppener, R. Maoz, and J. Sagiv, Nano Lett. 3, 761 (2003). 